\title{
ANGULAR MOMENTUM LOSS IN POST-MAIN SEQUENCE STELLAR EVOLUTION THROUGH THE PN STAGE
}

\author{
M. VILLATA \\ Istituto di Fisica Generale, Università di Torino, Via Pietro Giuria 1, I-10125 Torino, \\ Italy
}

A simple analytical model can explain the large angular momentum loss which a star suffers in its post-main-sequence evolution up to the white dwarf stage.

Mass and angular momentum losses in the PN stage are a well-established fact. Indeed it is known that white dwarfs are very slow rotators compared with what would be expected if the initial angular momentum of the parent main-sequence stars were conserved (see, e.g., Hardorp 1974; Greenstein et al. 1977; Weidemann 1977; Pilachowski and Milkey 1987; Koester and Herrero 1988). Thus very effective transport of angular momentum from the deep layers towards the surface has to be postulated, and the star probably maintains solid-body rotation for a significant part of its post-main-sequence evolution.

The basic assumptions of the model are that main-sequence stars and white dwarfs rotate as rigid bodies and that the mass loss between the two stages is due to stellar winds. We neglect the possible braking effect of magnetic fields, so that our results for the angular momentum loss will be regarded as lower limits. Hence, at each stage of the post-main-sequence evolution, the angular momentum loss is given by (with the assumption of spherical symmetry)

$$
-\mathrm{d} J \equiv-\mathrm{d}\left(h M R^{2} \Omega\right)=\frac{2}{3} R^{2} \Omega(-\mathrm{d} M),
$$

where $h M R^{2}$ is the moment of inertia of the star of mass $M$ and radius $R$, and $\Omega$ is its angular velocity.

A theoretical lower limit to the total angular momentum loss between the main sequence ("i") and the white dwarf stage (" $\mathrm{f}$ ") is given by the simple formula (Villata 1992)

$$
\frac{J_{\mathrm{f}}}{J_{\mathrm{i}}}=\left(\frac{M_{\mathrm{f}}}{M_{\mathrm{i}}}\right)^{\delta}, \quad \text { with } \quad \delta=\frac{2}{3} \frac{\ln \left(h_{\mathrm{f}} / h_{\mathrm{i}}\right)}{h_{\mathrm{f}}-h_{\mathrm{i}}},
$$

and turns out to be very large, varying from $99 \%$ for a star which evolves into a 0.5- $M_{\odot}$ white dwarf to more than $99.99 \%$ for a final mass higher than $1.1 M_{\odot}$.

We find that the theoretical upper limit to the rotational velocity of white dwarfs is $60 \mathrm{~km} \mathrm{~s}^{-1}$, in very good agreement with observational values.

\section{References}

Greenstein, J.L., Boksenberg, A., Carswell, R., and Shortridge, K. (1977), ApJ 212, 186.

Hardorp, J. (1974), A\&A 32, 133.

Koester, D. and Herrero, A. (1988), ApJ 332, 910.

Pilachowski, C.A. and Milkey, R.W. (1987), PASP 99, 836.

Villata, M. (1992), MNRAS (in press)

Weidemann, V. (1977), A\&A 59, 411. 